\title{
Informationen für die Klinik
}

\section{Neue Produkte}

Infusionsbeutel aus Polyethylen

Kunststoffverpackungen finden in der Phar-

mazie eine breite Verwendung, unter ande-

rem auch für die Verpackung von Infusions-

lösungen.

Die Firma Fresenius bietet hierfür seit kurzem einen praxisgerechten und in Vortests erfolgreich erprobten Kunststoffbeutel aus reinem Polyethylen an, der eine Vielzahl der von Klinikern geforderten Anforderungen erfüll-t.

Der neue Medipur ${ }^{\circledR}$-Infusionslösungsbeutel hat folgende Eigenschaften:

Material: Polyethylen ist aufgrund seiner chemischen Struktur frei von Weichmachern, Stabilisatoren oder sonstigen $\mathrm{Zu}-$ sätzen;

Eigenschaften: wegen seiner flachen Form stapelfreundlich und damit platzsparend; transparent, und damit sind Sichtkontrollen möglich; eine zu vernachlässigende Wasserverdunstungsrate und daher stabile Lösungskonzentrationen; keinen Direktkontakt von Lösung und Stopfen, weil eine Spezialmembran dies verhindert;

Einsatzmöglichkeiten: geeignet für alle handelsüb lichen Infusionsgeräte, auch für Druckinfusionen;

Umweltfreundlich: Polyethylen verhält sich im Erdreich neutral und zerfällt bei Verbrennung in Kohlendioxid und Wasser.

Weitere Informationen bei Fresenius AG, Borkenberg 14, D-6370 Oberursel 1, Tel.

06171/60-1.

Doppelkammer-Fertigspritze

Die Krewel-Werke bieten ab sofort eine Lyo-Ject Doppelkammerfertigspritze mit di-rekt lyophilisiertem Wirkstoff alternativ zur bisherigen parenteralen Darreichungsform CircoMaren ${ }^{\circledR} 4 \mathrm{mg}$ an. Im Hinblick auf eine einfache, schnelle und sichere Anwendung wird damit 
eine Eindo-sis-Packung zur Verfügung gestellt, die selbst in schwierigen Situationen leicht zu handhaben ist.

Jubiläum

Am 1. Juni 1986 wird Dr. Bernd Braun, 40 Jahre Leiter für Forschung und Entwick-lung der B. Braun Melsungen AG, 80 Jahre. Der diplomierte Chemiker und promovierte Mediziner trat 1936 in das väterliche Unter-nehmen ein, übernahm die wissenschaftlich-technische Gesamtleitung und entwickelte

zusammen mit seinem Bruder Otto das Un-ternehmen zu einem der größten Krankenhausversorger der Bundesrepublik und Eu-ropas.

Den von ihm initiierten Kasseler Sympo-sien, die seit 1957 in jedem Jahr stattfinden, ist die Entwicklung und Einführung der parenteralen Infusionstherapie für die Behand-lung des pathologischen Wasser- und Elek-trolythaushaltes im menschlichen Körper, der Schockbehandlung, der künstlichen Er-nährung sowie der prä- und postoperativen Therapie zu verdanken. Der Altersjubilar gehört zu den Gründungs-mitgliedern der Akademie für medizinische Fortbildung, Kassel-Bad Nauheim. Er rief zusammen mit seinem Bruder Otto vor 20 Jahren die B.-Braun-Stiftung ins Leben und förderte die Entwicklung der Medizin u. a. durch die Stiftung des Jubiläumspreises der Dt. Gesellschaft für Chirurgie und des Karl-ThomasPreises der Gesellschaft für Anästhesie und Wiederbelebung.

Preisverleihungen

Albert-Fraenkel-Preis 1986

Der Fraenkel-Preis 1986 geht in die Schweiz: Priv.-Doz. Dr. Otto Hess, Zurich, nahm die von Boehringer Mannheim gestif-tete Auszeichnung anläßlich der Eröffnung der 52. Jahrestagung der Deutschen Gesellschaft für Herz- und Kreislaufforschung am 4. April im Mannheimer Rosengarten ent-gegen.

Tagungspräsident Prof. Dr. Bender (Minister) wies in seiner Laudatio insbesondere auf die experimentellen und klinischen Ar-beiten des Preisträgers auf dem Gebiet der Inneren Medizin und der Kardiologie hin. Seit 1981 ist Dr. Hess als Oberarzt an der Kardiologischen Abteilung der Medizini-schen Poliklinik des Universitätsspitals in Zurich tätig.

Der mit 10000 DM dotierte Fraenkel-Preis wird seit 1956 jährlich an jüngere Wissen-schaftler aus dem deutschen Sprachraum verliehen, die sich durch besondere For-schungsaktivitäten auf dem Gebiet Herz/ Kreislauf qualifiziert haben. Die Auszeichnung erinnert an Albert Fraenkel, der im Jahre 1906 die intravenöse Strophantin-The-rapie zur Behandlung von Herzinsuffizienz entwickelte.

Albert-Knoll-Preis

Der Kardiologe Prof. Dr. med. Bernhard Maisch, Universitätsklinik Würzburg, und sein Mitarbeiter Dr. med. Ulrich Lotze er-

hielten am 1. März 1986 den Albert-Knoll-Preis der Saarländisch-Pfälzischen Interni-stenGesellschaft. Die ausgezeichnete Arbeit trägt den Titel: «Humorale Immunreak-tion bei Reizleitungs- und Reizbildungsstö-rungen». Der mit 10000 DM dotierte Preis wird jährlich von dem Ludwigshafener Pharmaunternehmen Knoll AG für hervor-ragende Arbeiten auf dem Gebiet der Inneren Medizin gestiftet. Die Preisträger nah-men die Auszeichnung anläßlich des 30. Jahreskongresses der Saarländisch-Pfälzischen Internisten-Gesellschaft in Saarbrük-ken entgegen.

Veranstaltungshinweise

Jahrestagung 1986 der Deutschen Gesellschaft für Pädiatrische Kardiologie 
Am 29. und 30. 9. 1986 findet die Jahrestagung der Deutschen Gesellschaft für Päd-iatrische Kardiologie in Aachen statt. Ta-gungsort ist das Klinikum. Die Tagung behandelt die Diagnostik sowie internistische und chirurgische Behandlung (einschließlich Intensivbehandlung) von Kindern mit Herzerkrankungen. Ein Schwerpunkt wird die Echokardiografie sein. Teilnehmer der Tagung sind Kinderärzte, Kinderkardiologen, Intensivmediziner und Herzchirurgen aus der Bundesrepublik Deutschland, aus Österreich, aus der Schweiz, aus den Niederlanden und aus mehreren Ostblockländern. Im Rahmen der Tagung findet eine Industrieausstellung statt.

Weitere Informationen bei Prof. Dr. G. von Bernuth, Abteilung Kinderkardiologie der Medizinischen Fakultät, RWTH Aachen, Pauwelsstraße, D-5100 Aachen.

Medica Montreux

Vom 21. Juli - 1. August 1986 findet in Montreux/Schweiz die «Medica Montreux» statt. An diesem 13. Internationalen Semi-narkongreß für ärztliche Fortbildung wird wieder ein umfangreiches Programm gebo-ten. Veranstalter und Auskunft: MEDICA Deutsche Gesellschaft zur Förde-rung der Med. Diagnostik e.V., Löffelstr. 1, D-7000 Stuttgart 70, Tel. (0711) 763443 +761454 .

Verantwortlich für die Zusammenstellung: Frans H. M. Stroeks, Puchheim b. München 